ARTICLE

DOI: $10.1038 / s 41467-018-03876-8$

OPEN

\title{
Induction of muscle stem cell quiescence by the secreted niche factor Oncostatin M
}

Srinath C. Sampath ${ }^{1,2,3}$, Srihari C. Sampath 1,2,3, Andrew T.V. Ho ${ }^{1}$, Stéphane Y. Corbel ${ }^{1,2}$, Joshua D. Millstone (1) ${ }^{2}$, John Lamb², John Walker ${ }^{2}$, Bernd Kinzel ${ }^{4}$, Christian Schmedt ${ }^{2} \&$ Helen M. Blau ${ }^{1}$

The balance between stem cell quiescence and proliferation in skeletal muscle is tightly controlled, but perturbed in a variety of disease states. Despite progress in identifying activators of stem cell proliferation, the niche factor(s) responsible for quiescence induction remain unclear. Here we report an in vivo imaging-based screen which identifies Oncostatin $M(O S M)$, a member of the interleukin- 6 family of cytokines, as a potent inducer of muscle stem cell (MuSC, satellite cell) quiescence. OSM is produced by muscle fibers, induces reversible MuSC cell cycle exit, and maintains stem cell regenerative capacity as judged by serial transplantation. Conditional OSM receptor deletion in satellite cells leads to stem cell depletion and impaired regeneration following injury. These results identify Oncostatin $M$ as a secreted niche factor responsible for quiescence induction, and for the first time establish a direct connection between induction of quiescence, stemness, and transplantation potential in solid organ stem cells.

\footnotetext{
${ }^{1}$ Baxter Laboratory for Stem Cell Biology, Department of Microbiology and Immunology, Institute for Stem Cell Biology and Regenerative Medicine, Stanford University School of Medicine, Stanford, CA 94305, USA. ${ }^{2}$ Genomics Institute of the Novartis Research Foundation, San Diego, CA 92121, USA. ${ }^{3}$ Division of Musculoskeletal Imaging, Department of Radiology, University of California San Diego School of Medicine, San Diego, CA 92103, USA. ${ }^{4}$ Novartis Institutes for BioMedical Research, 4056 Basel, Switzerland. These authors contributed equally: Srinath C. Sampath, Srihari C. Sampath. These authors jointly supervised this work: Christian Schmedt, Helen M. Blau. Correspondence and requests for materials should be addressed to S.C.S. (email: ssampath@gnf.org) or to H.M.B. (email: hblau@stanford.edu)
} 
S tem cells respond to tissue-specific activating signals by proliferating and giving rise to both committed progenitors, as well as quiescent daughter cells. Muscle repair is mediated by resident muscle stem cells (MuSC; also known as satellite cells). In response to myofiber damage, satellite cells break from quiescence and give rise to committed progenitors known as myoblasts, which can fuse both with each other, as well as with the damaged myofibers. Importantly, a subset of activated satellite cells are then re-selected into the quiescent stem cell niche in order to maintain the stem cell pool ${ }^{1}$. This process is highly regulated, involving not only cell autonomous signaling pathways, but also extensive regulation by tissue resident stromal cells and invading inflammatory cell populations ${ }^{2}$. These regulatory pathways are critical, as defects in stem cell quiescence, activation, or self-renewal have been implicated in a variety of disease states including aging-associated sarcopenia, muscular dystrophy, and cancer cachexia ${ }^{3-6}$.

Significant progress has been made toward elucidating the complex extracellular cues arising from the circulation and microenvironment that govern the behavior of tissue resident MuSC. Among the known soluble growth factor/receptor pathways, the HGF pathway has been demonstrated to control "alerting" of satellite cells in response to remote injury ${ }^{7}$, inducing a state of metabolic activation and priming for cell cycle reentry. FGF likewise regulates satellite cell activation ${ }^{8}$, while a downstream negative regulator of FGF receptor tyrosine kinase signaling, Sprouty1, plays an important role in the acquisition of quiescence of activated satellite cells ${ }^{9}$. Growth factor signaling eventually impinges on the $\mathrm{p} 38 \alpha / \beta$ MAPK pathway, which both genetic and pharmacologic data suggests functions as an important regulator of stemness and proliferation ${ }^{6,10}$. Additional levels of regulation are imposed via Notch/Delta-dependent regulation of self-renewal ${ }^{11-13}$, Wnt-dependent control of myogenic fate ${ }^{14}$, and asymmetric division associated with differential Pax7 expression ${ }^{15}$.

In addition to the growth factor pathways described above, previous work has demonstrated the importance of circulating cytokines in regulating MuSC and myofiber function. The IL-6 pathway has been a particular focus given the upregulation of this pleiotropic cytokine following exercise $e^{16}$, as well as the aberrant regulation of the downstream JAK-STAT signaling pathway during aging and in other disease states ${ }^{17}$. Studies with $I L-6$ deficient animals indicated a role for this cytokine in satellite cell and myoblast proliferation during experimentally induced hypertrophy ${ }^{18}$. The IL-6 family member Leukemia Inhibitory Factor (LIF) has likewise been demonstrated to induce proliferation of human and mouse myoblasts ${ }^{19}$. Indeed, it has recently been reported that inhibition of STAT3-dependent signaling, which is activated by both LIF and IL-6, can rescue age-associated proliferative defects in mouse satellite cells ${ }^{20}$ and promote satellite cell expansion ${ }^{21}$. Interpretation of the results of genetic and pharmacologic STAT3 inhibition is complicated, however, by the fact that this effector can be activated by numerous upstream signals, including both IL-6 and other cytokines $^{22}$.

Despite this progress, the precise signals governing the choice between quiescence and proliferation remain poorly understood, and this constitutes a major barrier to reversing the regenerative block seen in a variety of disease states. In particular, the identities of the factors regulating quiescence of adult stem cells have remained elusive. While bioinformatic analyses have begun to address this issue by identifying genes enriched in quiescent stem cells $^{23,24}$, the functional significance of most of these putative regulators has not been established. To address this deficiency, we have undertaken a systematic screen for secreted and transmembrane protein regulators of stem cell proliferation and engraftment. Employing an unbiased in vitro/in vivo imaging based screening strategy, we identify Oncostatin $\mathrm{M}$, a member of the IL-6 family of cytokines, as a novel regulator of stem cell quiescence, engraftment, and muscle regeneration.

\section{Results}

A secretomic screen for muscle stem cell regulators. We hypothesized that a secreted regulator of stem cell quiescence would be capable of suppressing MuSC activation and proliferation in vitro, while conversely maintaining the ability of these cells to engraft following transplantation in vivo. To identify such regulators, we designed and carried out a protein library screen on purified MuSCs, using a combination of in vitro and in vivo imaging to distinguish between effects on stem cell proliferation and engraftment (Fig. 1a). Primary muscle stem cells isolated from luciferase transgenic mice ${ }^{25,26}$ were cultured on uncoated plastic plates in the presence of pools of proteins derived from the GNF Secretomics library, which contains purified versions of known or predicted mammalian secreted proteins, as well as the extracellular domains of known or predicted single-pass transmembrane proteins $^{27}$. No additional growth factors (e.g., FGF) were added. In vitro bioluminescence imaging (BLI) was used to assess stem cell proliferation (Supplementary Fig. 1a, top), followed by cell harvesting and transplantation into the tibialis anterior (TA) muscle of hindlimb-irradiated recipients to assess engraftment, a sensitive measure of regeneration (Supplementary Fig. 1a, bottom). Serial in vivo imaging was performed over a timecourse to quantify engraftment activity of the transplanted cells, as indicated by a progressive increase in light output, a feature of stem cells but not committed progenitors ${ }^{25}$. A comparison was made to both untreated cells, as well to known inducers of stem cell proliferation, FGF and the p38 MAPK inhibitor, SB2026,10. Protein pools demonstrating increased engraftment on serial in vivo imaging were iteratively deconvolved through rounds of subpool rescreening to identify the single protein responsible for the engraftment activity (Fig. 1b).

Pooled screening of $\sim 600$ predominantly mouse proteins yielded 5 hits in two phenotypic classes. The first class was exemplified by a pool driving both in vitro proliferation, as well as in vivo engraftment (pool A; Fig. 1c, Supplementary Fig. 1b). Iterative deconvolution identified the responsible protein as FGF2, a known mitogenic factor for $\mathrm{MuSC}^{28}$, providing strong validation of the assay. The second phenotypic class exemplified the hypothesized behavior for an inducer of reversible stem cell quiescence, driving little in vitro stem cell proliferation but robust in vivo engraftment. Deconvolution of one such pool (pool Y; Fig. 1c, Supplementary Fig. 1b) identified the extracellular domain of the TNFa receptor (Supplementary Fig. 1c), which acts as a receptor decoy for TNFa and which is consistent with the known pro-differentiation function of TNFa on satellite cells ${ }^{29}$. Pools $\mathrm{F}$ and $\mathrm{H}$ were not able to be completely deconvolved, possibly due to partial contribution of multiple protein factors. Strikingly, deconvolution of the remaining pools in this phenotypic class identified the same protein in three differentially epitope-tagged forms, Oncostatin M (OSM; pools D, E, and S; Fig. 1c, star; Supplementary Fig. 1b). No engraftmentpromoting activity was seen in subpools lacking Oncostatin M (Supplementary Fig. 1d, e). Recombinant OSM obtained from commercial sources demonstrated similar engraftment-inducing activity (Fig. 1d). Histological analysis of engrafted recipient mice confirmed the presence of donor-derived myofibers (Supplementary Fig. 2a), in keeping with the good correlation between BLI and histology seen previously 6,25 .

Single cell RT-PCR analysis of purified MuSC demonstrated expression of the Oncostatin $M$ receptor (OSMR $\beta$ ) within a 
a

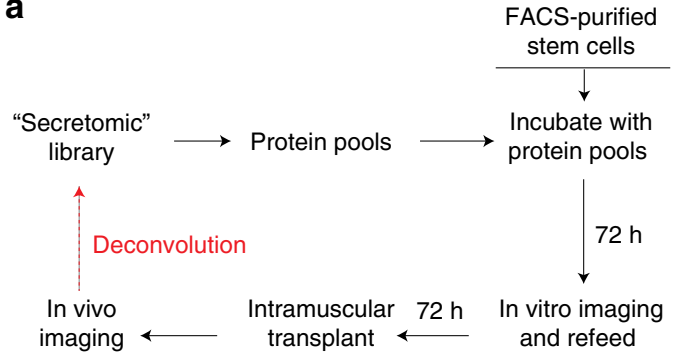

b

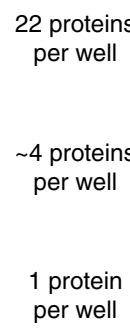

8
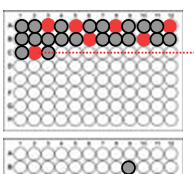

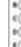
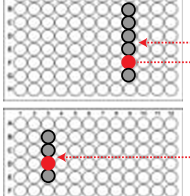

Round 1 deconvolution

Round 2 deconvolution
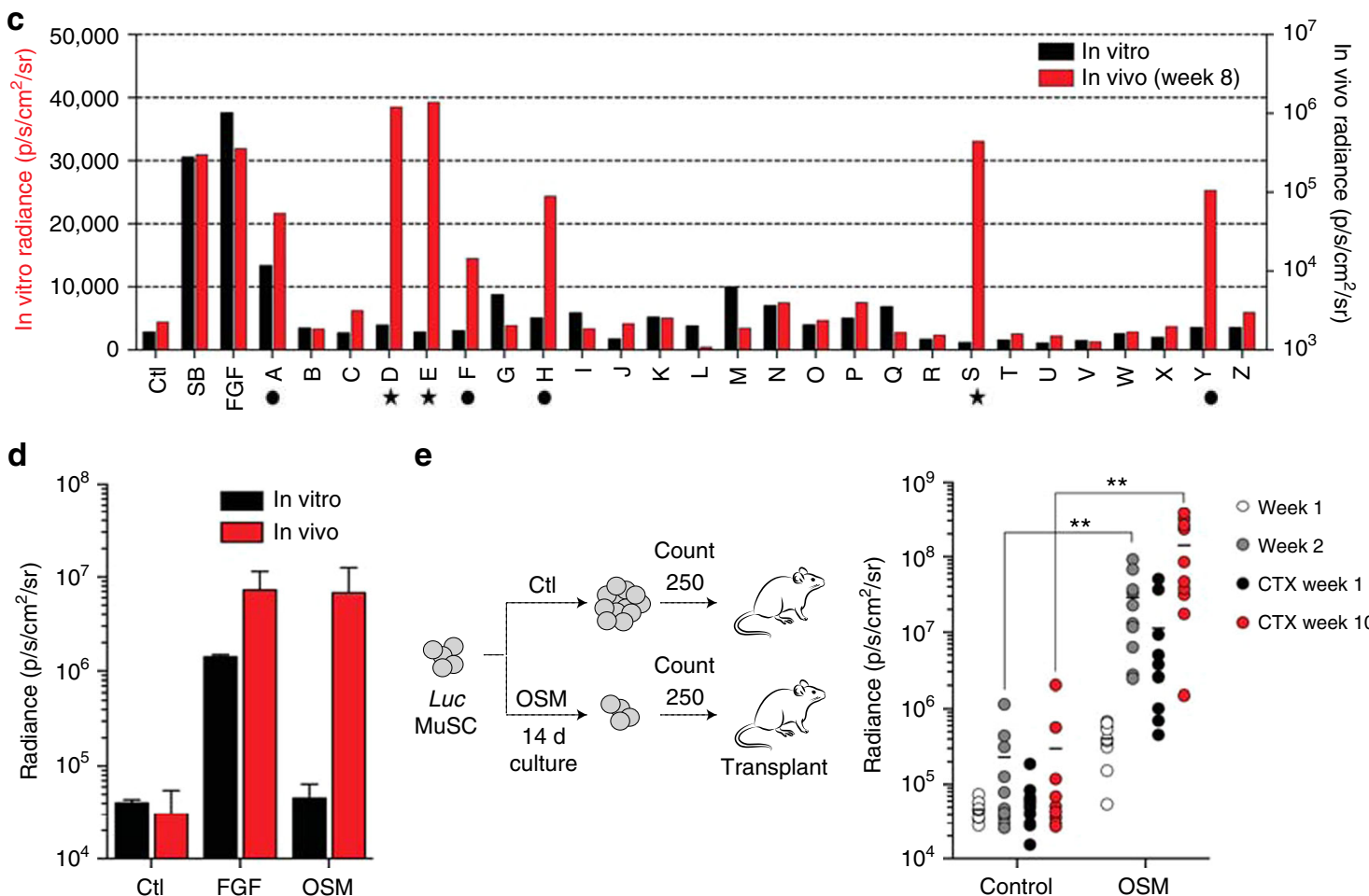

e
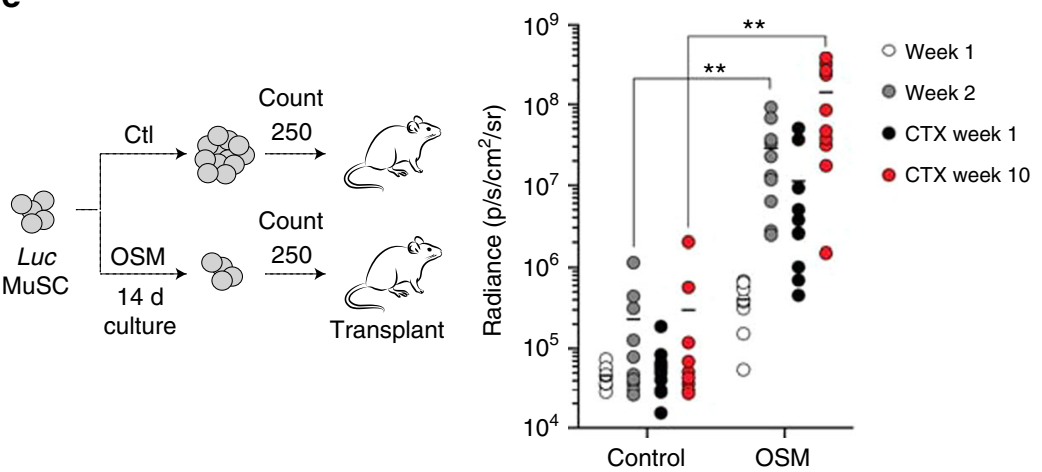

Fig. 1 Identification of Oncostatin $\mathrm{M}$ as a potent inducer of muscle stem cell engraftment. a Schematic of combined in vitro/in vivo bioluminescence imaging (BLI) screening strategy. $\mathbf{b}$ Schematic of deconvolution for positive protein pools. Pools of 22 proteins scoring for engraftment in the initial round of screening were iteratively deconvolved into pools of 4 proteins, and then individual proteins. c Results of multiplexed in vitro/in vivo protein screening. Black, results of in vitro BLI; red, results of in vivo BLI. Ctl: control buffer; SB: SB202190; FGF: recombinant human basic fibroblast growth factor. Symbols below the axis mark pools containing OSM (star), and all other hits (circle; includes FGF, TNFR, and incompletely deconvolved pools). d Representative BLI results following treatment of 800 FACS-sorted MuSC with either FGF or Oncostatin M (OSM; $100 \mathrm{ng} / \mathrm{mL}$ ) for 6 days in vitro, followed by intramuscular transplantation (3 8-12 week old animals each) and in vivo BLI measurement 6 days (open circles) or 2 weeks (shaded circles) after transplant. e Schematic (left) demonstrating transplantation of 250 counted progeny of $\mathrm{CD}_{11 \mathrm{~b}}{ }^{-} \mathrm{Sca} 1^{-} \mathrm{CD} 31^{-} \mathrm{CD} 45^{-} \mathrm{CD} 34^{+} \alpha \alpha 7$ integrin ${ }^{+}$stem cells following long-term 2 week culture with OSM control conditions (FGF). Serial BLI following transplantation of counted cells (right). Each point indicates an individual hindlimb that received a 250 cell transplant, $n=10$ per condition. BLI is shown for weeks 1 (white) and 2 (light gray) pre-CTX, and weeks 1 (dark gray) and 10 (red) postCTX. Double asterisks indicate $p<0.01$ in comparison with control condition at the same timepoint

subset comprising $\sim 20 \%$ of Pax $7^{+}$cells (Supplementary Fig. 2b), which may reflect either true heterogeneity, or limited sensitivity of the single cell detection method. To exclude effects on proliferation as a confounding variable, stem cell engraftment was next determined on a per-cell basis. Satellite cells were cultured for 2 weeks either with OSM or in standard medium containing FGF, and intramuscular transplant of 250 counted cells was then performed (Fig. 1e, left). Serial in vivo imaging revealed progressive increase of light output, indicative of engraftment, and injury of the stem cell-engrafted muscle with cardiotoxin led to a drop in BLI signal followed by a robust rebound, confirming the presence of functional satellite cells (Fig. 1e, right). BLI signal was significantly higher in muscle receiving OSM-treated MuSC, consistent with increased per-cell engraftment and regeneration.
Regulation of muscle stem cell quiescence by OSM. To further investigate the anti-proliferative effect of OSM, we performed serial in vitro BLI. This demonstrated inhibition of stem cell proliferation at all timepoints (Fig. 2a, Supplementary Fig. 3a), which was attributable to decreased S-phase entry (Fig. 2b). Proliferation of stem cells activated by culture on muscle fibers ex vivo was also strongly inhibited (Supplementary Fig. 3b, c), and OSM treatment of primary mouse myoblasts likewise decreased levels of the M-phase marker phospho-H3S10 (Fig. 2c). Previous studies have demonstrated a critical role of the cyclin-dependent kinase inhibitor p27/Kip1 in quiescence of stem cells both in muscle, as well as other lineages ${ }^{30,31}$. We also observed a significant decline in p27 transcript in cultured MuSC, and moreover found that OSM treatment 
a

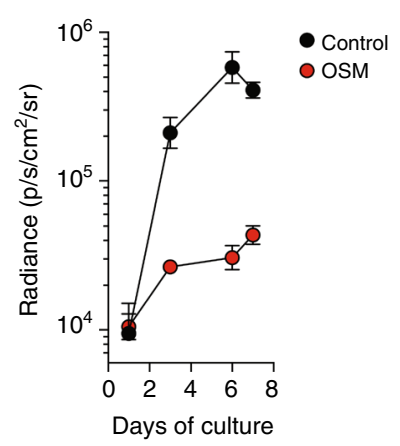

b

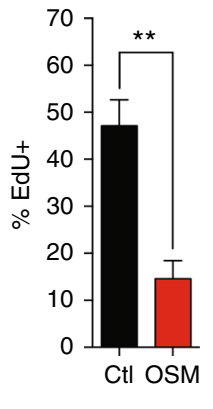

C

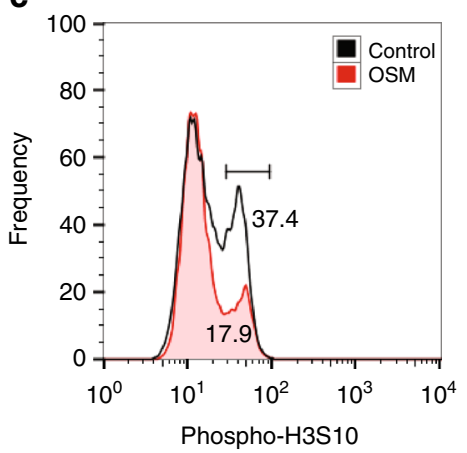

d

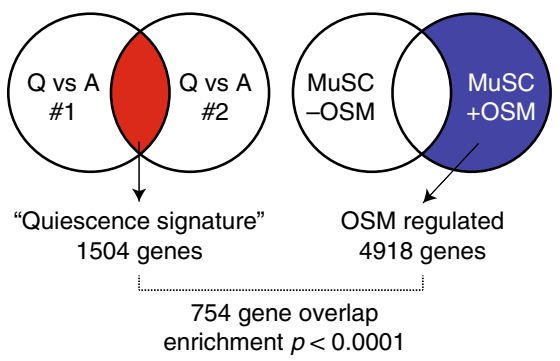

e

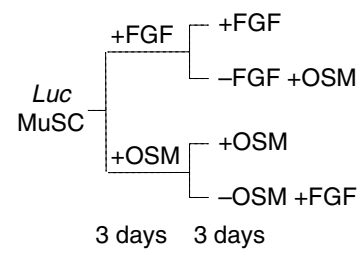

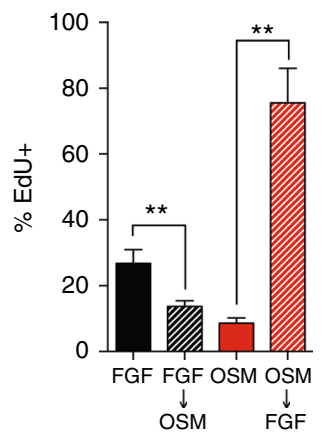

Fig. 2 OSM induces reversible cell cycle exit and a molecular signature of quiescence. a FACS-sorted muscle stem cells purified from luciferase transgenic mice were cultured for 7 days at 2500 sorted counts per well $\left(\sim 31\right.$ cells $\left./ \mathrm{mm}^{2}\right)$ in the presence (closed circle) or absence (open circles) of OSM, as well as low dose FGF $(0.5 \mathrm{ng} / \mathrm{ml})$. Serial in vitro BLI was performed at the indicated timepoints. Mean \pm SD is shown, $n=4$ replicates. b FACS-sorted MuSC cultured for 6 days as in (A) were pulsed with EdU for the last $12 \mathrm{~h}$ of culture. Mean \pm SD is shown, $n=4$ replicates. c Induction of cell cycle exit in myoblasts in response to OSM. Primary myoblasts were cultured for 3 days in growth medium with (red) out without (gray) added OSM. Cells were fixed, intracellular staining performed for mitotic H3S10 phosphorylation, and analyzed by flow cytometry. d Diagram demonstrating overlap between a bioinformatically-derived gene expression "quiescence signature" for satellite cells, and genes regulated during OSM-treatment of muscle stem cells in vitro. OSM regulation data was derived mean of 2 biological replicates. e Schematic of switch experiment to demonstrate reversibility of OSM-induced cell cycle exit (left). FACS-sorted MuSC were cultured for 6 days under the indicated conditions, and then pulsed with EdU for the final $12 \mathrm{~h}$ of culture (right). Mean $\pm S D$ is shown, $n=4$ replicates; greater than 300 cells were counted per replicate

maintained $p 27$ expression at levels seen in freshly isolated, noncultured stem cells (Supplementary Fig. 4a), suggesting that OSM might directly induce quiescence. To explore this possibility, we compared the global transcriptional profile of freshly isolated MuSCs to MuSCs cultured for 3 days with or without OSM, as well as to prior transcriptional studies of muscle stem cell quiescence $^{23,24}$. The published analyses were used to extract a transcriptional signature of quiescence regulated genes. A comparison of this "quiescence signature" with the transcriptional response to OSM revealed highly significant enrichment (Fig. 2d), which was independently confirmed by qPCR analysis (Supplementary Fig. 4b). Over half of the signature genes were present within the OSM-regulated set (Supplementary Data 1), consistent with induction of a global transcriptional quiescence program.

Reversibility is the hallmark feature of quiescence, and we therefore tested whether OSM-induced cell cycle exit was reversible by culturing satellite cells in either activating (-OSM) or non-activating (+OSM) conditions for 3 days, after which the medium conditions were switched and EdU pulse labeling performed (Fig. 2e, left). Non-cycling OSM-treated cells robustly re-entered cell cycle following stimulation, as measured by both EdU incorporation (Fig. 2e, right) and cell proliferation (Supplementary Fig. 4c); This anti-proliferative effect on satellite cells was dependent on MEK activity (Supplementary Fig. 4d), consistent with prior reports of OSM activity in other cell types 32,33 .
OSM treatment maintains stemness in vitro. Although both satellite cells and their committed progeny (myoblasts) express Pax7 and can contribute to myofiber formation ${ }^{34}$, only satellite cells are capable of mediating serial regeneration following injury ${ }^{25}$. The ability to reisolate and retransplant engrafted satellite cells is thus a strict test of stem cell identity, and we therefore tested whether OSM-treated MuSC fulfill these criteria. We employed a newly generated reporter allele in which an internal ribosome entry site and green fluorescent protein (IRES-GFP) are inserted into the $3^{\prime}$ untranslated region of the Pax7 locus (Pax7 GFP; Supplementary Fig. 5a). This allele specifically labels $\mathrm{CD} 34^{+} / \alpha 7$ integrin $^{+}$satellite cells in skeletal muscle (Supplementary Fig. 5b). MuSC from Pax ${ }^{\mathrm{GFP}}$ mice were cultured in the presence or absence of OSM, and intramuscular transplant of equal numbers of treated stem cells was performed. Despite high initial engraftment of cells cultured either with or without OSM (Fig. 1d), Pax $7^{\mathrm{GFP}}$-expressing MuSC could only be re-isolated from mice engrafted with OSM-treated cells (Fig. 3a). To establish that the engrafted cells represent bona fide functional stem cells, $\mathrm{Pax} 7^{\mathrm{GFP}+}$ cells were re-isolated from primary recipients of OSM-treated MuSC, and 50 counted cells were transferred into the hindlimbs of irradiated secondary recipients. BLI demonstrated engraftment in $\sim 40 \%$ of recipients, and cardiotoxin injury induced initial loss followed by rebound of BLI signal, a pattern characteristic of stem cell-mediated regeneration (Fig. 3b). Previous studies have demonstrated that such increased serial transplantation ability corresponds with higher Pax7 
a

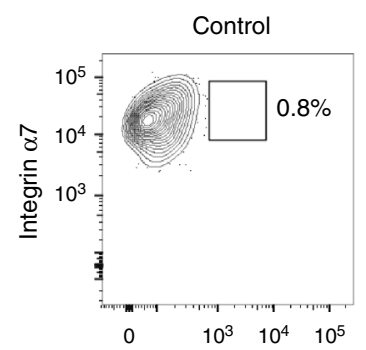

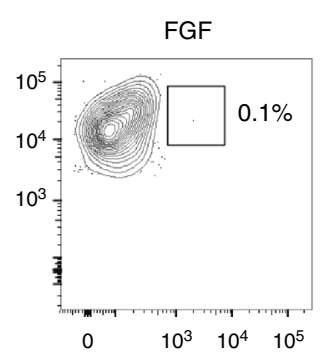

Pax7-GFP

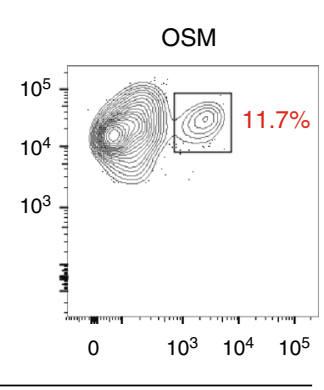

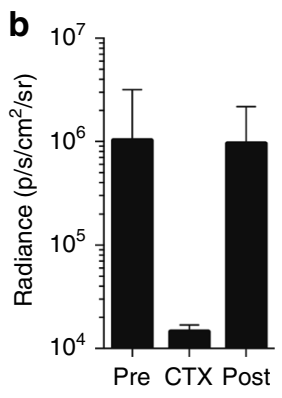

C

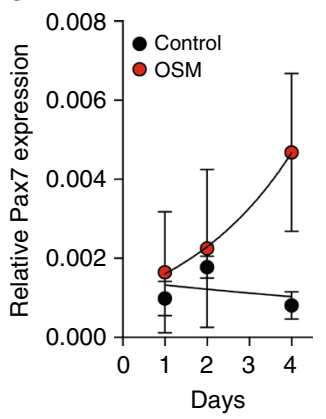

d

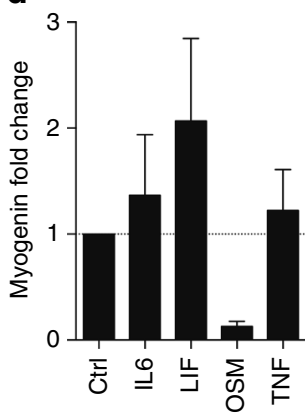

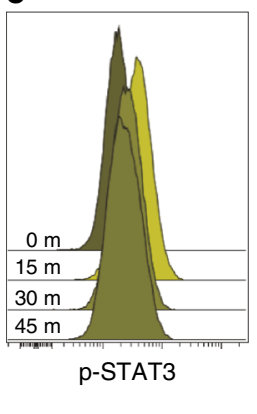

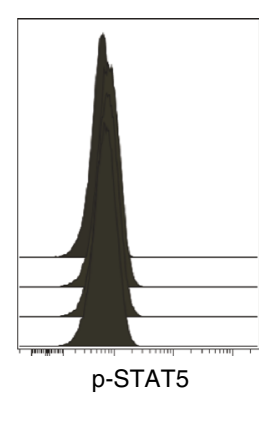

f

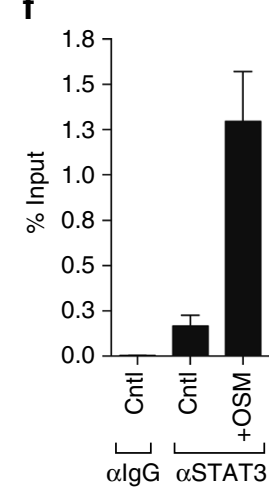

Fig. 3 OSM treatment enhances stem cell serial transplantation and inhibits differentiation. a FACS analysis of stem cells reisolated from primary transplant recipients receiving either control (left), FGF (middle), or OSM-treated (right) stem cells. Only the live, $\mathrm{CD}_{11 \mathrm{~b}}{ }^{-} \mathrm{Sca} 1^{-} \mathrm{CD} 31^{-} \mathrm{CD} 45^{-} \mathrm{CD} 34^{+} \alpha 7$ integrin ${ }^{+}$stem cell population is shown. b MuSCs (CD11b-Sca1 ${ }^{-} \mathrm{CD} 31^{-} \mathrm{CD} 45^{-} \mathrm{CD} 34^{+} \alpha 7$ integrin ${ }^{+} \mathrm{GFP}^{+}$) were reisolated from OSM primary transplant mice as described in $\mathbf{a}$, transplanted equally into 5 limbs (secondary transplant recipients; 50 MuSC/limb; 8-12 week old recipients), and monitored by serial BLI. At day 25 following transplant, cardiotoxin (CTX) was injected to induce myofiber damage and stimulate stem cell expansion and tissue regeneration. The average radiance at each timepoint is indicated, and demonstrates regenerative capacity (BLI signal loss followed by rebound) of serially transplated OSM-treated MuSC. Percentage of engrafted mice was $40 \%$ both pre and post-CTX injury ( $2 / 5$ total mice). Note that untreated cells give no engraftment, and no MuSC are therefore present for reisolation/transplant. c qRT-PCR analysis of Pax7 expression following 6 day culture of FACS-sorted MuSC in the presence of OSM (gray circles) or buffer control (black). Mean \pm SD is shown, $n=3$ replicates. $\mathbf{d}$ qRT-PCR analysis of myogenin expression following 6 day culture of FACS-sorted MuSC with the indicated cytokines and growth factors. Expression was normalized to GAPDH expression. Mean \pm SD is shown, $n=3$ replicates. e Timecourse intracellular flow cytometry of primary mouse myoblasts treated for the indicated period of time with OSM, and stained for either phospho-STAT3 (left) or phospho-STAT5 (right). 0 (unstimulated), 15, 30, and 45 min timepoints are shown following cytokine addition. Frequencies are represented as histograms. $\mathbf{f}$ ChIP performed using primary myoblasts cultured for 6 days either with or without OSM. Immunoprecipitation was performed with either control IgG or STAT3-specific antibodies, and PCR was performed using primers specific for a predicted STAT3 binding site adjacent to the Pax7 locus

levels ${ }^{15}$, and we likewise found that OSM treatment not only induced $\operatorname{Pax} 7$ (Fig. 3c) but also reciprocally decreased expression of the differentiation marker myogenin (Supplementary Fig. 6a). This effect was unique among the related IL-6 family of cytokines (Fig. 3d $)^{20,21}$. Intracellular staining and flow cytometry in primary myoblasts demonstrated that OSM preferentially activated STAT3 rather than STAT5 (Fig. 3e). Analysis of the Pax7 locus revealed a previously uncharacterized binding site for STAT3, and chromatin immunoprecipitation (ChIP) from primary myoblasts confirmed direct occupancy of this site by STAT3 in response to OSM treatment (Fig. 3f). Taken together, these data reveal a MEK and STAT3-mediated response to OSM in satellite cells, leading to quiescence induction as well as maintenance of stem cell identity and function.

Functions of OSM signaling in vivo. To address the endogenous source of OSM in muscle, we assessed protein levels in muscle tissue and found they were highest in the resting state, decreased rapidly following injury, and reappeared beginning at day 4 and robustly by day 10 , concomitant with myofiber regeneration (Fig. 4a). This suggests that OSM is produced in a paracrine fashion from the myofiber niche, rather than in an autocrine fashion from satellite cells. Moreover, this timecourse is inconsistent with immune cells, such as macrophages, being the primary source of OSM, as reported by others in the heart ${ }^{33}$. Immunofluorescence staining of OSM in uninjured muscle indeed revealed localization within myofibers of uninjured muscle, both around myonuclei and the fiber periphery (Fig. 4b), consistent with OSM production by myofibers as a secreted niche factor. To investigate the physiological significance of nicheinduced OSM signaling, we derived mice deficient for the obligate OSM receptor, OSMR $\beta$ (Supplementary Fig. 6b). Intermediate OSMR $\beta$ levels were present in heterozygous animals (Supplementary Fig. 6b), consistent with previously reported haploinsufficiency ${ }^{33}$. EdU incorporation was significantly higher in OSMR-deficient satellite cells (Supplementary Fig. 6c), demonstrating that OSM signaling in satellite cells restrains stem cell proliferation. Importantly, OSMR $\beta$ was required for the proquiescence effect of OSM (Supplementary Fig. 6c), excluding the possibility of additional receptors mediating this signal.

OSMR-deficient mice demonstrated no difference in stem cell number or myofiber cross-sectional area in the steady state (Supplementary Fig. 6d, e); this is in keeping with the reappearance of OSM protein during myofiber repair post injury (Fig. 4a), and consistent with a role in re-establishment of quiescence following activation. Serial injury of OSMR-deficient muscle would therefore be expected to deplete the satellite cell 
a
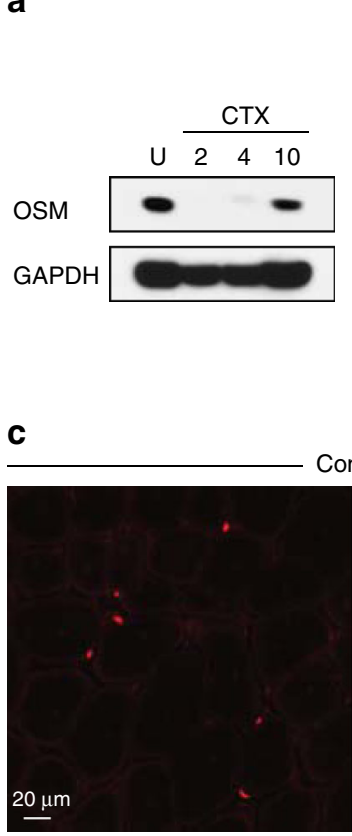

$\operatorname{Pax} 7$ b

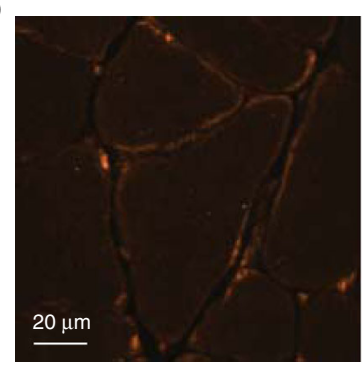

OSM

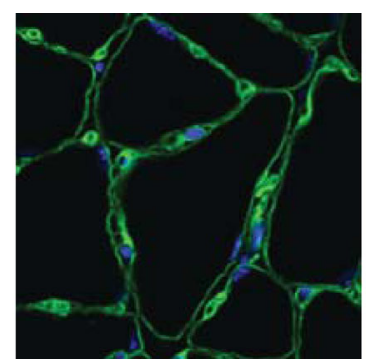

Laminin DAPI

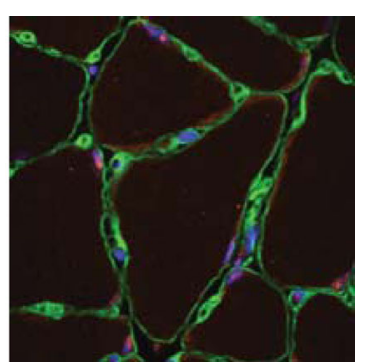

Merge

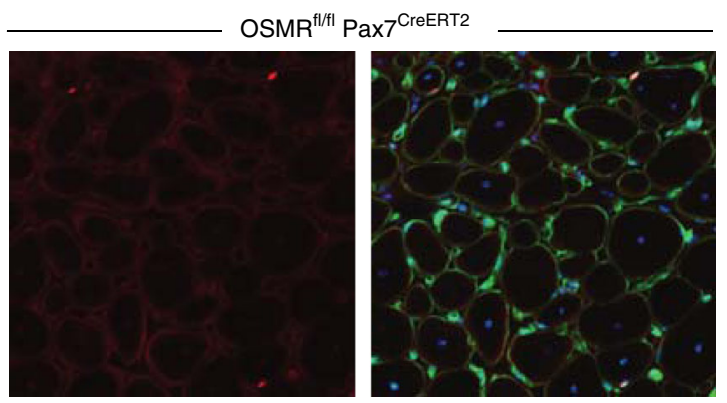

Pax7

Pax7 Laminin DAPI

e

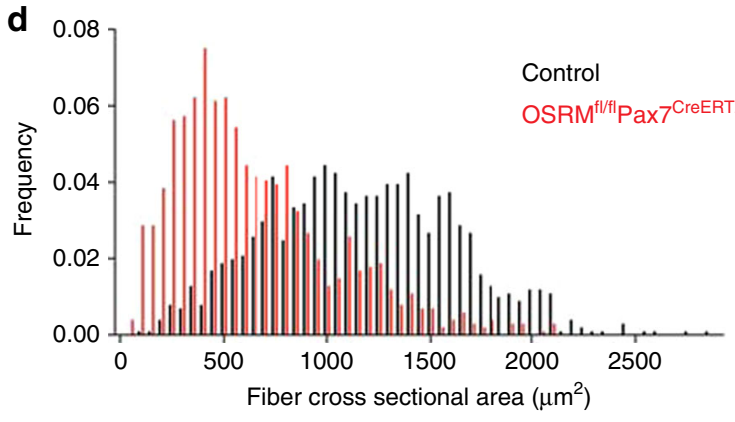

f

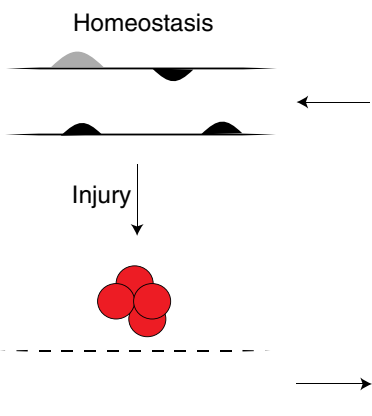

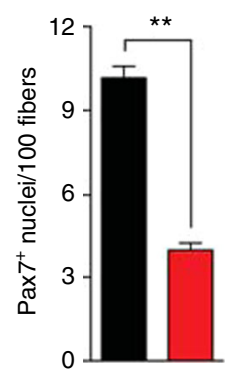

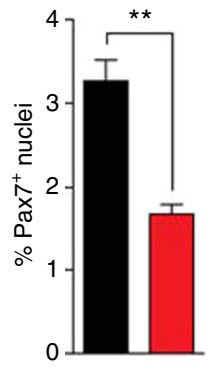

Control

OSMR ${ }^{\mathrm{f} / \mathrm{t} \mid}$

$\mathrm{Pax} 7$ CreERT2

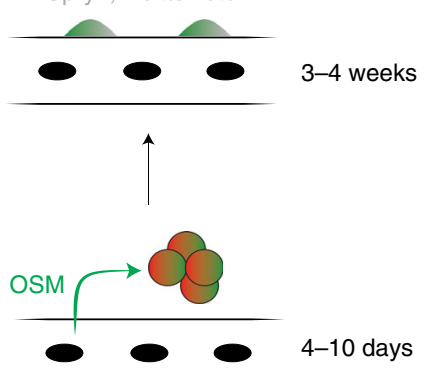

Fig. 4 OSM signaling regulates quiescence induction and is required for proper muscle regeneration. a Western blot of tibialis anterior muscle, either uninjured $(U)$ or at the indicated days following cardiotoxin injury. b Immunofluorescence analysis of wild type uninjured TA muscle. Blue: DAPI; Green: Laminin; Red: OSM. Note the expected perinuclear staining pattern of OSM, consistent with synthesis and export through the known perimyonuclear ER/ Golgi network ${ }^{49,50}$. c Representative immunofluorescence images from tibialis anterior muscle of control (Osmr ${ }^{\mathrm{fl} /+}$ ) or conditional $\mathrm{knockout}\left(\mathrm{Osmr}^{\mathrm{fl} / \mathrm{fl}}\right.$ Pax $7^{\text {CreERT2 }}$ ) animals following deletion and 2 rounds of cardiotoxin injury and regeneration. For each sample, single channel Pax 7 staining is shown on the left and a merged 3 channel image is shown on the right. Blue: DAPI; Green: Laminin; Red: Pax7. d Cross-sectional area (CSA) distribution of myofibers from TA muscle of control (Osmr ${ }^{\mathrm{fl} /+}$ ) or conditional knockout (Osmr ${ }^{\mathrm{fl} / \mathrm{fl}} \mathrm{Pax} 7^{\mathrm{CreERT2}}{ }^{2}$ ) animals (3 8-12 week old animals each) following deletion and 2 rounds of cardiotoxin injury and regeneration. $>1000$ fibers were quantified per genotype. e Quantitation of Pax $7^{+}$stem cells in serial sections from tibialis anterior muscle of control (-Cre, Osmr ${ }^{\mathrm{fl} /+}$ ) or conditional knockout ( $+\mathrm{Cre}, \mathrm{Osmr} \mathrm{fl}^{\mathrm{fl} \mid} \mathrm{Pax} 7^{\mathrm{CreERT} 2}$ ) animals following deletion and 2 rounds of cardiotoxin injury and regeneration. Results are plotted as Pax $7^{+}$nuclei per 100 fibers (left) or Pax $7^{+}$nuclei as a percentage of all visualized nuclei (right). $\mathbf{f}$ Model for OSM mediated quiesence induction during skeletal muscle regeneration. Under homeostatic conditions, stem cells (gray) are deeply quiescent but can become activated and proliferate (red) following injury. As regenerated myofibers begin to form, secretion of OSM (green) allows quiescence induction by actively promoting cell cycle exit within a subset of activated satellite cells while also maintaining stem cell identity. At later timepoints, 2-3 weeks following injury, upregulation of intrinsic regulators such as Sprouty1 matures the re-selected satellite cell pool back toward the deeply quiescent homeostatic state 
pool. Indeed, two rounds of cardiotoxin injury in OSMR $\beta$ deficient mice resulted in a substantial decrease in satellite cell numbers (Supplementary Fig. 7a), associated with a marked regenerative block and $>50 \%$ reduction in mean myofiber crosssectional area (CSA; Supplementary Fig. 7b, c, d). To exclude that this reflected effects of OSM on other cell populations such as inflammatory cells, we used Cre-loxP mutagenesis to conditionally ablate OSMR $\beta$ in $\mathrm{Pax}^{+}$MuSC using inducible lineagerestricted Cre expression ${ }^{35}$. Serial muscle injury of conditionally deleted animals led to impaired muscle regeneration, marked decrease in myofiber cross sectional area, and severe depletion of satellite cells (Fig. 4c-e; Supplementary Fig. 7e). The regenerative defect of satellite-cell specific OSMR deletion was indistinguishable from that of whole-body constitutive deletion, demonstrating a specific requirement for OSM signaling in satellite cells.

\section{Discussion}

Here we describe a novel in vivo imaging-based screen which identifies Oncostatin $\mathrm{M}$ as a key niche-derived secreted regulator of muscle stem cell quiescence. OSM is, to our knowledge, one of only two niche-localized factors known to induce quiescence in solid organ stem cells ${ }^{36}$, and the first shown to maintain transplantation potential. The ability of OSM to promote MuSC engraftment without inducing proliferation is distinct from previously reported factors such as regulators of $\mathrm{p} 38 \mathrm{MAPK}^{6,10}$, as well as related cytokines such as IL-6 and LIF (Fig. $2 \mathrm{~h})^{18,20,21,37}$. The quiescence-inducing function of OSM in skeletal muscle is likewise distinct from its effect in other tissues such as the heart, where it acts to induce cardiomyocyte proliferation ${ }^{33}$, and more closely resembles the cytostatic effect originally described in cancer cell lines and mouse myoblasts ${ }^{38-40}$. This cytostatic effect was moreover shown to be associated with STAT3 activation, and inhibition of CDK2 activity. Although OSM was paradoxically reported to have a pro-proliferative effect on human myogenic progenitor cells, interpretation of this finding is complicated by the fact that human (but not mouse) OSM also signals through the proliferation-inducing LIF receptor (LIFR) pathway ${ }^{41,42}$.

The temporal expression pattern of myofiber-derived OSM resembles that of Sprouty1, a satellite cell-intrinsic inhibitor of FGF signaling whose re-expression following injury is likewise critical for re-induction of stem cell quiescence ${ }^{9}$. Importantly, while OSM is re-expressed by fibers within 4-10 days of injury, Sproutyl re-expression requires 3 weeks ${ }^{9}$. We suggest that this difference may define an early stage of quiescence, in which nascent myofibers secrete OSM in order to induce cell cycle exit and maintain stem cell identity in a subset of activated satellite cells (Fig. 4f). This model is in keeping with restricted expression of OSMR within a subset of satellite cells (Supplementary Fig. 2b), and adds to our knowledge of heterogeneity within the stem cell pool $^{43,44}$. In addition to its role following muscle injury, we speculate that OSM signaling may play additional roles in the steady state, for instance preventing full activation of stem cells induced by remote damage to enter the " $\mathrm{G}_{\text {Alert }}$ " state ${ }^{7}$. Future work will address this possibility, the fate of OSMR deficient MuSC following activation, as well as whether dysregulation of OSM levels with aging or chronic injury may contribute to impaired maintenance and function of the stem cell pool ${ }^{3,4,6,28}$. It will likewise be important to understand the mechanisms by which signaling pathways shared by multiple IL-6 family members are translated into distinct biological responses ${ }^{44,45}$. Finally, we anticipate that the in vivo imaging-based screening approach reported here will prove useful in identifying additional niche components controlling the function of satellite cells and stem cells in other lineages.

\section{Methods}

Mice. Experimental protocols involving mice were reviewed and approved by the Stanford University Administrative Panel on Laboratory Animal Care and the GNF Institutional Animal Care and Use Committee. All experiments were performed in compliance with institutional guidelines. All experiments utilized adult (8-12 week old) female mice. Hindlimb-irradiated NOD/SCID mice (Jackson Laboratory) were used as stem cell recipients during screening of protein library pools. Subsequent experiments involving stem cell transplants utilized hindlimbirradiated, immunocompromised NSG mice. Ubiquitously expressing transgenic CAG-luciferase-GFP mice were obtained and maintained as described previously $^{6,25}$. Lineage reporter Pax7-IRES-GFP mice were generated using a strategy similar to one previously described ${ }^{46}$. In brief, to generate a targeting vector for homologous recombination, Pax7 genomic sequences were amplified from C57BL/ 6 mouse genomic DNA and Pax7 homology arms were cloned into a targeting vector containing IRES-EGFP and FRT-flanked neomycin cassettes. C57BL/6 mouse ES cells were transfected by electroporation of linearized Pax7 targeting vector. Transfected ES cells were selected for neomycin resistance using $0.2 \mathrm{mg} / \mathrm{ml}$ Geneticin (Invitrogen \#10131-019). After 10 days of transfection, 300 G418resistant ES cell clones were isolated and genotyped by PCR for homologous recombination with primers CTATCGCCTTCTTGACGA (neomycin) and GTGTCCCTGGTGTCTTGT (Pax7). Correct targeting was confirmed by Southern blot using a neomycin-specific probe, which allowed the exclusion of random integration events of the targeting vector. Selected targeted ES cells were injected into BALB/c blastocysts and chimeric mice were bred with $\mathrm{C} 57 \mathrm{BL} / 6$ females to obtain Pax7-IRES-GFP knock-in mice. To eliminate the FRT-flanked neomycin cassette, Pax7 gene targeted mice were crossed with a mouse line expressing Flp recombinase and analyzed for the loss of the neomycin cassette. Pax7-GFP mice without neo were used for further analysis.

The Osmr allele was obtained from Jax (B6;129-Osmrtm1.1Nat/J) and typed via real time PCR (Transnetyx). For complete deletion, floxed mice were bred to Thy 1 - cre to induce deletion in the germline. The deleted allele was confirmed by PCR and bred away from the Cre driver. The strain was maintained by intercross of heterozygous deleted animals. For conditional deletion, the floxed allele was bred to $\mathrm{Pax} 7^{\mathrm{CreERT} 235}$. To induce deletion, Osmr ${ }^{\mathrm{fl} / \mathrm{fl}} \mathrm{Pax} 7^{\mathrm{CreERT} 2}$ mice or controls (floxed homozygous or heterozygous without Cre driver) were injected on five consecutive days with $200 \mu \mathrm{l}$ of $20 \mathrm{mg} / \mathrm{ml}$ tamoxifen in corn oil (Sigma), and then subsequently maintained on tamoxifen-containing chow (TD.130855, Harlan-Teklad) in order to prevent counterselection against deleted satellite cells ${ }^{47}$.

Stem cell purification and culture. Mouse muscle stem cells were purified essentially as previously described ${ }^{6}$. In brief, tibialis anterior and gastrocnemius muscles were dissected and digested with Collagenase II and Dispase (Worthington Biochemical Corporation) for a total of 90-120 min. Following centrifugation and resuspension, the cell suspension was strained a $40-\mu \mathrm{m}$ filter (BD Biosciences). Cells were stained with the following biotinylated antibodies: anti-CD45 (BD Biosciences, clone 30F11, catalog \# 553078, 1:500), anti-CD11b (BD Biosciences, clone M1/70, catalog \# 553309, 1:800), anti-CD31 (eBiosciences, clone 390, catalog \# 13-0311-82, 1:200) and anti-Scal (BD Biosciences, clone E13-161.7, catalog \# 553334, 1:200). Cells were then incubated with streptavidin (SA) microbeads (Miltenyi Biotech), SA-Texas Red (Invitrogen, catalog \# S872, 1:200), PE labeled anti-integrin $\alpha 7$ antibody labeled with phycoerythrin (AbLab, clone R2F2, catalog \# 10ST215, 1:500) and anti-CD34 eFluor660 (eBioscience, clone RAM34, catalog \# 50-0341-82, 1:67). In some experiments, cells were stained with eFluor 450 directly conjugated lineage antibodies (anti-CD45, anti-CD31, antiScal, anti-CD11b, anti-Ter119; eBioscience). Dead cells were stained with propidium iodide. Cells sorting on a FACS Aria 2 routinely yielded $>95 \%$ CD $34^{+} \alpha 7$ integrin $^{+}$cells from a single round of sorting. Scatter plots were analyzed using FlowJo (TreeStar). All in vitro culture experiments were performed using media changes with fresh recombinant protein added every 3 days. For screening, Histagged cDNA clones comprising the GNF Secretomics Collection were expressed in 293 T Freestyle cells and affinity purified using nickel agarose using in-house automation instrumentation. Retained proteins were eluted in TBS with $250 \mathrm{mM}$ imidazole and were pooled manually. Pooled proteins were added to 800 purified stem cell counts at $10 \%$ final culture volume; no attempts were made to normalize the concentration of individual proteins within the pools, which therefore varied as a function of protein expression and elution efficiency. Media was changed after 3 days and fresh protein-containing media was added for another 3 days prior to transplant. MuSC were cultured under minimal conditions on plastic without exogenous extracellular matrix, conditions that favor commitment and differentiation ${ }^{48}$. Standard muscle stem cell culture was performed in DMEM/F10 (50:50), 15\% FBS, $1 \%$ penicillin-streptomycin, and $2.5 \mathrm{ng} / \mathrm{ml} \mathrm{rh}$ bFGF. For screening experiments, culture was performed in medium above but without FGF. Pilot experiments indicated that use of collagen coated plates dramatically reduced assay window, therefore uncoated plastic plates were used throughout, including for long-term (14 day) stem cell culture. Recombinant Oncostatin M (R\&D Systems), recombinant human basic FGF (Promega), SB202190 (EMD Chemicals) and U0126 (Cell Signaling) were obtained commercially. Surface area for experiments performed in chamber slides (Fig. 2a) was $0.8 \mathrm{~cm}^{2}$, with an initial seeding density of $\sim 31$ cells $/ \mathrm{mm}^{2}$ 
Muscle stem cell transplantation and muscle injury. Stem cell harvesting, intramuscular injection, and cardiotoxin-mediated muscle injury were all performed essentially, as previously described ${ }^{6}$, with the exception that $50 \mathrm{ul} 10 \mu \mathrm{M}$ cardiotoxin was used for all injuries. For counted cell transplants, stem cells were counted manually using a hemocytometer prior to transplant.

Bioluminescence imaging. Bioluminescence imaging (BLI) was performed using either a Xenogen 100 or IVIS Spectrum CT, as previously described ${ }^{6,25,48}$. Briefly, $150 \mu \mathrm{L}$ D-luciferin (reconstituted at $30 \mathrm{mg} / \mathrm{mL}$ in sterile water; Caliper LifeSciences) was administered by intraperitoneal injection into anesthetized mice. BLI were acquired using $60 \mathrm{~s}$ exposures for a total of $12 \mathrm{~min}$ following luciferin injection. Images were recorded and analyzed using Living Image software (Caliper LifeSciences). For images acquired on the Xenogen 100, a signal value of 100,000 photons per second was routinely used to define the engraftment threshold, as previously described ${ }^{6,25}$. Engraftment thresholds were $\sim 10$-fold higher for imaging performed on the IVIS Spectrum CT, reflecting the higher sensitivity on this instrument and intrinsic detector characteristics between machines. In vitro imaging was performed similarly but with a 1:1000 final dilution of stock luciferin.

Graphical and statistical analysis. Graphs and statistics were generated using GraphPad Prism software. Student's two-tailed $t$ tests were used to calculate $p$ values.

Cell and tissue staining. Tibialis anterior (TA) muscle sections were prepared for staining essentially as previously described ${ }^{6,48}$. For OSMR staining, whole TA muscles were first fixed with $0.5 \%$ paraformaldehyde/PBS for $2 \mathrm{~h}$ at $4 \mathrm{C}$, followed by incubation in $20 \%$ sucrose/PBS overnight at 4 C. $10 \mu \mathrm{M}$ sections were cut for all stainings. Antibodies were: anti-laminin (Millipore, catalog $\# 05^{-} 206,1: 250$ ), antiGFP (Invitrogen, catalog \#A11122, 1:200), anti-OSMR (R\&D, catalog \#AF665, 1:200), anti-Pax7 (Santa Cruz Biotechnology, catalog \#sc81648, 1:50; or Developmental Studies Hybridoma Bank, $2 \mu \mathrm{g} / \mathrm{mL}$ final concentration), AlexaFluor 594-conjugated donkey anti-rat IgG1 and AlexaFluor 488-conjugated donkey antirabbit (Jackson ImmunoResearch, catalog \# 712-585-150 and 711-545-152 respectively, 1:200 each). Nuclei were counterstained with either DAPI (Invitrogen) or TO-PRO-3 (Invitrogen). Images were acquired with an AxioPlan2 epifluorescent microscope (Carl Zeiss) with ORCA-ER digital camera (Hamamatsu Photonics).

Single-cell RT-PCR. Single cell RT-PCR was performed, as described ${ }^{6,25}$. In brief, MuSCs were obtained from digested muscle as above, and three rounds of FACS sorting was performed in order to obtain a highly purified $\mathrm{CD} 34^{+} \alpha 7$ integrin ${ }^{+}$ MuSC population. In the last sorting step, the cells were dispensed into PCR tubes containing lysis buffer under single cell conditions (1 cell/well) or non-single cell conditions ( 50 cells/well). Multiplexed RT-PCR was performed using the following primers: Pax7 ext FWD GAGTTCGATTAGCCGAGTGC; Pax7 ext REV GGTTAGCTCCTGCCTGCTTA; Pax7 int FWD GCGAGAAGAAAGCCAA ACAC; Pax7 int REV GGGTGTAGATGTCC GGGTAG Myf5 ext FWD AGACGCCTGAAGAAGGTCAA; Myf5 ext REV AGCTGGACACGGAGCTTT TA; Myf5 int FWD C CACCAACC CTAAC CAGAGA; Myf5 int REV CTGTTCTTTCGGGACCAG AC; Myod1 ext FWD TACCCAAGGTGGAGAT CCTG; Myod1 ext REV GTGGAGATGCGCTCCACTAT; Myod1 int FWD GCC TTCTACGCACCTGGAC; Myod1 int REV ACTCTTCCCTGCCTGGACT

EdU labeling. For in vitro labeling experiments, satellite cells were treated with 10 $\mu \mathrm{M}$ 5-ethynyl-2'-deoxyuridine for the last $12 \mathrm{~h}$ of culture. Cells were fixed, permeabilized, and EdU incorporation detected using the Click-iT EdU Alexa Fluor 488 Imaging Kit (Life Technologies) per manufacturer's protocol.

qRT-PCR. Analysis of purified cultured satellite cells was performed, as described $^{6,25}$. RNA was isolated using the RNeasy Micro Kit (Qiagen), and reverse transcribed using SuperScript III. RT-PCR was performed using a SYBR Green PCR Master Mix (Applied Biosystems). Quantification was performed using the $2-\Delta \Delta \mathrm{Ct}$ method and normalized to Gapdh. Primer sequences were as follows: p27/Kip1 REV CCGGGCCGAAGAGATTTCTG; OSMR FWD CATCCCGAAGCGAAGTCT TGG; OSMR REV GGCTGGGACAGTCCATTCTAAA; GAPDH FWD AGGTCGGTGTGAACGGATTTG; GAPDH REV TGTAGACCATGTAGT TGAGGTCA; Pax7 FWD TCTCCAAGATTCTGTGCCGAT; Pax7 REV CGGGGTTCTCTCTCTTATACTCC.

ChIP-qPCR. Stat3-ChIP was performed following the standard protocol from the ChIP-IT Express Enzymatic kit (Active Motif, California) on chromatin isolated from primary myogenic progenitor cells $\left(8 \times 10^{6}\right)$ cultured with the same culture media and condition as described for muscle stem cells. Briefly, cells were crosslinked for 10 min with $1 \%$ formaldehyde and quenched with $12.5 \mathrm{mM}$ glycine for 5 min. Nuclei were obtained by incubating with $1 \mathrm{ml}$ hypertonic lysis buffer and protease and phosphatase inhibitors as supplied for $30 \mathrm{~min} 4{ }^{\circ} \mathrm{C}$ and releasing with 20 strokes through a dounce homogenizer on ice. Chromatin was obtained with the nuclear digestion buffer and fragmented to 300-100 base-pairs with the enzymatic shearing cocktail in $50 \%$ glycerol as provided by the kit for $12 \mathrm{~min}$ at $37^{\circ} \mathrm{C} .10 \%$ of the sheared chromatin was saved as an input control, and 10 ug of sheared chromatin from each condition were immunoprecipitated with antibody to mouse STAT3 (610190, BD Bioscience) or normal mouse IgG (Cell Signaling) overnight at $4{ }^{\circ} \mathrm{C}$, followed by $1 \mathrm{~h}$ of incubation at $4{ }^{\circ} \mathrm{C}$ with Protein G-coated magnetic beads (Active Motif). Washing and reverse crosslinking was performed according to manufacturer's protocol. The precipitated DNA was amplified and analyzed by real-time SYBR Green PCR (Applied Biosystem) on an ABI PRISM $7900 \mathrm{HT}$ Sequence Detection system (Applied Biosystem). Primer pairs (EpiTect GPM105088(+)01A, SA Bioscience) designed for quantitative RT-PCR analysis were used to amplify the putative Stat3 binding site adjacent to the mouse Pax7 locus (Chr4: 139387970-139388390), identified via Biobase Transfac analysis (Biobase Corp. MA). The Stat3 occupancy was calculated as a ratio of the amplification efficiency of Stat3 ChIP over input chromatin.

Intracellular FACS. Cells were fixed with $4 \%$ formaldehyde for $10 \mathrm{~min}$ at RT and permeabilized by adding cell suspension drop-wise into ice-cold $100 \%$ methanol while gentle vortexing to a final concentration of $90 \%$ methanol and incubate on ice for $30 \mathrm{~min}$. Permeabilized cells were resuspended in FACS buffer (1xPBS, 1\% BSA), stained with antibody for $30 \mathrm{~min}$ at room temperature and analyzed via FACS Calibur flow cytometer (BD, Bioscience). Phospho-Histone H3 (ser10) was performed using the Alexa Fluor 488 conjugated H3S10 primary antibody (9708, Cell Signaling). Phospho-Stat 3 and Phospho-Stat5 were performed using PEmouse anti-Stat3 (Y705) (612569, BD Bioscience) and PerCP-Cy5.5 mouse antiStat5 (pY694) (560118, BD Bioscience) respectively.

Microarray analysis. Total RNA was prepared with Qiagen RNeasy kits. In total $20 \mathrm{ng}$ of total RNA was prepared for array analysis using Affymetrix $3^{\prime}$ IVT Express amplification reagents. In total $15 \mathrm{ng}$ of cRNA was hybridized to Affymetrix MOE_430_2 arrays, and arrays were washed, stained, and scanned using standard methods. CEL files were processed using MAS5. Microarray data have been submitted to GEO (accession number GSE69976).

Bioinformatic analysis. OSM signature genes were defined as the top-quartile of the most up-regulated genes using the ratio of OSM-treatment/no-OSM treatment Similarly a quiescence signature was defined using published data ${ }^{23,24}$ as the intersection of the top quartile of up-regulated genes (ratio of quiescence vs activated) in two comparisons. These two signature sets were then compared to each other and the number of overlapping genes was found to be higher than expected by chance as assessed by Fisher's Exact Test ( $n=754$ genes, 2.01 fold enrichment, $p=4 \mathrm{e}-105)$.

Data availability. Microarray data are available via GEO (accession number GSE69976). The data that support the findings of this study are available on request from the corresponding authors (S.C.S., H.M.B.).

Received: 24 May 2017 Accepted: 16 March 2018 Published online: 18 April 2018

\section{References}

1. Yin, H., Price, F. \& Rudnicki, M. A. Satellite cells and the muscle stem cell niche. Physiol. Rev. 93, 23-67 (2013).

2. Aurora, A. B. \& Olson, E. N. Immune modulation of stem cells and regeneration. Cell Stem Cell 15, 14-25 (2014).

3. Sousa-Victor, P. et al. Geriatric muscle stem cells switch reversible quiescence into senescence. Nature 506, 316-321 (2014).

4. Sacco, A. et al. Short telomeres and stem cell exhaustion model Duchenne muscular dystrophy in mdx/mTR mice. Cell 143, 1059-1071 (2010).

5. He, W. A. et al. NF-kappaB-mediated Pax7 dysregulation in the muscle microenvironment promotes cancer cachexia. J. Clin. Investig. 123, 4821-4835 (2013).

6. Cosgrove, B. D. et al. Rejuvenation of the muscle stem cell population restores strength to injured aged muscles. Nat. Med. 20, 255-264 (2014).

7. Rodgers, J. T. et al. mTORC1 controls the adaptive transition of quiescent stem cells from G0 to G(Alert). Nature 510, 393-396 (2014).

8. Yablonka-Reuveni, Z., Seger, R. \& Rivera, A. J. Fibroblast growth factor promotes recruitment of skeletal muscle satellite cells in young and old rats. $J$ Histochem. Cytochem.: Off. J. Histochem. Soc. 47, 23-42 (1999).

9. Shea, K. L. et al. Sproutyl regulates reversible quiescence of a self-renewing adult muscle stem cell pool during regeneration. Cell Stem Cell 6, 117-129 (2010).

10. Bernet, J. D. et al. p38 MAPK signaling underlies a cell-autonomous loss of stem cell self-renewal in skeletal muscle of aged mice. Nat. Med. 20, 265-271 (2014).

11. Conboy, I. M., Conboy, M. J., Smythe, G. M. \& Rando, T. A. Notch-mediated restoration of regenerative potential to aged muscle. Science 302, 1575-1577 (2003).

12. Kuang, S., Kuroda, K., Le Grand, F. \& Rudnicki, M. A. Asymmetric self-renewal and commitment of satellite stem cells in muscle. Cell 129, 999-1010 (2007). 
13. Bjornson, C. R. et al. Notch signaling is necessary to maintain quiescence in adult muscle stem cells. Stem Cells 30, 232-242 (2012).

14. Brack, A. S. et al. Increased Wnt signaling during aging alters muscle stem cell fate and increases fibrosis. Science 317, 807-810 (2007).

15. Rocheteau, P., Gayraud-Morel, B., Siegl-Cachedenier, I., Blasco, M. A. \& Tajbakhsh, S. A subpopulation of adult skeletal muscle stem cells retains all template DNA strands after cell division. Cell 148, 112-125 (2012).

16. Hiscock, N., Chan, M. H., Bisucci, T., Darby, I. A. \& Febbraio, M. A. Skeletal myocytes are a source of interleukin- 6 mRNA expression and protein release during contraction: evidence of fiber type specificity. FASEB J.: Off. Publ. Fed. Am. Soc. Exp. Biol. 18, 992-994 (2004).

17. Hunter, C. A. \& Jones, S. A. IL-6 as a keystone cytokine in health and disease. Nat. Immunol. 16, 448-457 (2015).

18. Serrano, A. L., Baeza-Raja, B., Perdiguero, E., Jardi, M. \& Munoz-Canoves, P. Interleukin-6 is an essential regulator of satellite cell-mediated skeletal muscle hypertrophy. Cell Metab. 7, 33-44 (2008).

19. Austin, L., Bower, J., Kurek, J. \& Vakakis, N. Effects of leukaemia inhibitory factor and other cytokines on murine and human myoblast proliferation. $J$. Neurol. Sci. 112, 185-191 (1992).

20. Price, F. D. et al. Inhibition of JAK-STAT signaling stimulates adult satellite cell function. Nat. Med. 20, 1174-1181 (2014).

21. Tierney, M. T. et al. STAT3 signaling controls satellite cell expansion and skeletal muscle repair. Nat. Med. 20, 1182-1186 (2014).

22. Wang, X., Crowe, P. J., Goldstein, D. \& Yang, J. L. STAT3 inhibition, a novel approach to enhancing targeted therapy in human cancers (review). Int. J. Oncol. 41, 1181-1191 (2012)

23. Fukada, S. et al. Molecular signature of quiescent satellite cells in adult skeletal muscle. Stem Cells 25, 2448-2459 (2007).

24. Pallafacchina, G. et al. An adult tissue-specific stem cell in its niche: a gene profiling analysis of in vivo quiescent and activated muscle satellite cells. Stem Cell Res. 4, 77-91 (2010).

25. Sacco, A., Doyonnas, R., Kraft, P., Vitorovic, S. \& Blau, H. M. Self-renewal and expansion of single transplanted muscle stem cells. Nature 456, 502-506 (2008).

26. Cao, Y. A. Shifting foci of hematopoiesis during reconstitution from single stem cells. Proc. Natl Acad. Sci. USA 101, 221-226 (2004).

27. Gonzalez, R. et al. Screening the mammalian extracellular proteome for regulators of embryonic human stem cell pluripotency. Proc. Natl Acad. Sci. USA 107, 3552-3557 (2010).

28. Chakkalakal, J. V., Jones, K. M., Basson, M. A. \& Brack, A. S. The aged niche disrupts muscle stem cell quiescence. Nature 490, 355-360 (2012).

29. Palacios, D. et al. TNF/p38alpha/polycomb signaling to Pax7 locus in satellite cells links inflammation to the epigenetic control of muscle regeneration. Cell Stem Cell 7, 455-469 (2010).

30. Chakkalakal, J. V. et al. Early forming label-retaining muscle stem cells require p27kip1 for maintenance of the primitive state. Development 141, 1649-1659 (2014).

31. Zou, P. et al. p57(Kip2) and p27(Kip1) cooperate to maintain hematopoietic stem cell quiescence through interactions with Hsc70. Cell Stem Cell 9, 247-261 (2011).

32. Song, H. Y. et al. Oncostatin $M$ decreases adiponectin expression and induces dedifferentiation of adipocytes by JAK3- and MEK-dependent pathways. Int. J. Biochem. \& Cell Biol. 39, 439-449 (2007).

33. Kubin, T. et al. Oncostatin $\mathrm{M}$ is a major mediator of cardiomyocyte dedifferentiation and remodeling. Cell Stem Cell 9, 420-432 (2011).

34. Rando, T. A., Pavlath, G. K. \& Blau, H. M. The fate of myoblasts following transplantation into mature muscle. Exp. Cell Res. 220, 383-389 (1995).

35. Murphy, M. M., Lawson, J. A., Mathew, S. J., Hutcheson, D. A. \& Kardon, G. Satellite cells, connective tissue fibroblasts and their interactions are crucial for muscle regeneration. Development 138, 3625-3637 (2011).

36. Delgado, A. C. et al. Endothelial NT-3 delivered by vasculature and CSF promotes quiescence of subependymal neural stem cells through nitric oxide induction. Neuron 83, 572-585 (2014).

37. Spangenburg, E. E. \& Booth, F. W. Multiple signaling pathways mediate LIFinduced skeletal muscle satellite cell proliferation. Am. J. Physiol. Cell Physiol. 283, C204-C211 (2002).

38. Zarling, J. M. et al. Oncostatin M: a growth regulator produced by differentiated histiocytic lymphoma cells. Proc. Natl Acad. Sci. USA 83, 9739-9743 (1986).

39. Xiao, F. et al. Oncostatin $M$ inhibits myoblast differentiation and regulates muscle regeneration. Cell Res. 21, 350-364 (2011).

40. Kim, H., Jo, C., Jang, B. G., Oh, U. \& Jo, S. A. Oncostatin M induces growth arrest of skeletal muscle cells in G1 phase by regulating cyclin D1 protein level. Cell Signal 20, 120-129 (2008).

41. Latroche, C. et al. Coupling between myogenesis and angiogenesis during skeletal muscle regeneration is stimulated by restorative macrophages. Stem Cell Rep. 9, 2018-2033 (2017).
42. Heinrich, P. C., Behrmann, I., Muller-Newen, G., Schaper, F. \& Graeve, L. Interleukin-6-type cytokine signalling through the gp130/Jak/STAT pathway. Biochem J. 334, 297-314 (1998).

43. Tierney, M. T. \& Sacco, A. Satellite cell heterogeneity in skeletal muscle homeostasis. Trends Cell Biol. 26, 434-444 (2016).

44. Blau, H. M., Cosgrove, B. D. \& Ho, A. T. The central role of muscle stem cells in regenerative failure with aging. Nat. Med. 21, 854-862 (2015)

45. Hirahara, K. et al. Asymmetric action of STAT transcription factors drives transcriptional outputs and cytokine specificity. Immunity 42, 877-889 (2015).

46. Keller, C., Hansen, M. S., Coffin, C. M. \& Capecchi, M. R. Pax3:Fkhr interferes with embryonic Pax3 and Pax7 function: implications for alveolar rhabdomyosarcoma cell of origin. Genes Dev. 18, 2608-2613 (2004).

47. von Maltzahn, J., Jones, A. E., Parks, R. J. \& Rudnicki, M. A. Pax7 is critical for the normal function of satellite cells in adult skeletal muscle. Proc. Natl Acad. Sci. USA 110, 16474-16479 (2013).

48. Gilbert, P. M. et al. Substrate elasticity regulates skeletal muscle stem cell selfrenewal in culture. Science 329, 1078-1081 (2010).

49. Percival, J. M. \& Froehner, S. C. Golgi complex organization in skeletal muscle: a role for Golgi-mediated glycosylation in muscular dystrophies? Traffic 8, 184-194 (2007)

50. Percival, J. M. et al. rAAV6-microdystrophin rescues aberrant Golgi complex organization in mdx skeletal muscles. Traffic 8, 1424-1439 (2007).

\section{Acknowledgements}

This report is dedicated to the memory of Matt Furia. We thank Penney Gilbert and Ben Cosgrove for advice and methodological input; John Joslin, Sarah Cox, Mark Knuth, Tony Orth, Heath Klock, and Scott Lesley for the Secretomics library; Angela Christiano for the OSM staining protocol; Mary Frazer, Richard Eddins, Kassie Koleckar, and Peggy Kraft for animal husbandry support; and Richard Glynne for advice and support. The Stanford Center for Innovation in In-Vivo Imaging (SCI3), the Stanford Shared FACS Facility (SSFF), the Stanford Lokey Stem Cell Research FACS Facility, and the Stanford Veterinary Service Center (VSC) are thanked for technical support. This study was supported by the Baxter Foundation, NIH R01 AG020961, NIH R01 NS089533 and CIRM R5-07469 and the Li Ka Shing Foundation (to H.M.B.)

\section{Author contributions}

Experiments were designed, carried out, and interpreted by S.C.S., S.C.S., A.T.V.H., S.Y. C., J.M., C.S., and H.M.B. J.L. and J.W. contributed to microarray experiments and bioinformatics analysis. Pax7-GFP mice were developed by B.K. All authors contributed to writing and revision of the manuscript.

\section{Additional information}

Supplementary Information accompanies this paper at https://doi.org/10.1038/s41467 018-03876-8.

Competing interests: S.C.S., S.C.S., S.Y.C., J.M., J.L., J.W., B.K., and C.S. are employees of the Genomics Institute of the Novartis Research Foundation and the Novartis Institutes for BioMedical Research. The remaining authors declare no competing interests.

Reprints and permission information is available online at http://npg.nature.com/ reprintsandpermissions/

Publisher's note: Springer Nature remains neutral with regard to jurisdictional claims in published maps and institutional affiliations.

Open Access This article is licensed under a Creative Commons Attribution 4.0 International License, which permits use, sharing, adaptation, distribution and reproduction in any medium or format, as long as you give appropriate credit to the original author(s) and the source, provide a link to the Creative Commons license, and indicate if changes were made. The images or other third party material in this article are included in the article's Creative Commons license, unless indicated otherwise in a credit line to the material. If material is not included in the article's Creative Commons license and your intended use is not permitted by statutory regulation or exceeds the permitted use, you will need to obtain permission directly from the copyright holder. To view a copy of this license, visit http://creativecommons.org/ licenses/by/4.0/.

(C) The Author(s) 2018 